\title{
ORIGINAL
}

\section{ESTUDIO SEROLÓGICO DE AGRUPACIÓN DE CASOS DE HEPATITIS B EN SEIS FAMILIAS DE RAZA CHINA EN ALMERÍA}

\author{
Pilar Barroso García (1), Ma Angeles Lucerna Méndez (2), Estrella Adrián Monforte (3), Tesifón \\ Parrón Carreño (4) \\ (1) Sección de Epidemiología. Distrito Sanitario de Atención Primaria Levante-Alto Almanzora (Almería). \\ (2) Servicio de Medicina Preventiva. Hospital "La Inmaculada" Huércal-Overa (Almería). \\ (3) Consultorio de Mojácar Playa. Zona Básica de Salud de Vera (Almería). \\ (4) Servicio de Salud. Delegación Provincial de Salud de Almería.
}

\section{RESUMEN}

Fundamento: Tras la detección de dos casos de serología positiva para el virus de la hepatitis B en miembros de 6 familias chinas se inició un estudio de convivientes. El objetivo fue conocer la amplitud de la infección en el entorno familiar de los casos detectados.

Métodos: Estudio descriptivo. Población de estudio: 24 miembros de 6 familias de raza china Variables: Edad, sexo, diagnóstico serológico, factores de riesgo, actitud sanitaria. Fuentes de información: Historias clínicas, datos serológicos, encuesta epidemiológica y cartillas de vacunación. Se realizó un enfoque familiar y se utilizó el genograma. Análisis de datos: Distribución binomial para cálculo de probabilidad de ocurrencia del proceso a estudiar.

Resultados: Se estudió a 14 hombres $(58,3 \%)$ y 10 mujeres $(41,7 \%)$ con edades comprendidas entre 1 y 54 años. El grupo de edad con más sujetos estudiados fue el de 21-30 años (37,5\%). Se registraron 12 infecciones crónicas de hepatitis B $(50 \%)$. No se encontró relación con los factores de riesgo estudiados en la encuesta epidemiológica realizada. La probabilidad de ocurrencia de este número de hepatitis crónica fue de 0,066 × $10^{-6}$.

Conclusiones: Se concluye que probablemente la prevalencia de infección encontrada se debiera a transmisión intrafamiliar. Dada la baja probabilidad de ocurrencia de un proceso de estas características, se considera que la agrupación de casos encontrada es elevada.

Palabras clave: Hepatitis B, transmisión intrafamiliar, inmigrantes asiáticos, China.

Correspondencia:

Pilar Barroso García

Distrito Sanitario de Atención Primaria Levante-Alto Almanzora

C / Silvestre Martínez de Haro s/n

Huércal-Overa

04600 Almería

Correo electrónico: mariap.barroso.sspa@juntadeandalucia.es
ABSTRACT

\section{Hepatitis B Case Grouping Serological Study among Six Chinese Families in Almeria, Spain}

Background: Following the detection of two cases of members of 6 Chinese families having tested positive for the hepatitis B virus, a study of those living in these families was begun for the purpose of knowing the spread of the infection within the family environment of the cases detected.

Methods: Descriptive study. Population under study: 24 members of six Chinese families. Variables: Age, sex, serological diagnosis, risk factors, healthcare-related attitude. Information sources: Clinical records, serological data, epidemiological survey and immunization cards. A family focus was employed and the genogram used. Data Analysis: Distribution Binomial spread for calculating probability of occurrence of the process to be studied.

Results: A total of 14 males (58.3\%) and 10 females (41.7\%) ranking from 1 to 54 years of age were studied. The age group having the largest number of subjects studied was the age 21-30 group (37.5\%). Twelve chronic hepatitis $B$ infections were recorded (50\%). No relationship was found to exist with the risk factors studied in the epidemiological survey conducted. The probability of this number of chronic hepatitis cases occurring was $0.066 \times 10^{-6}$.

Conclusions: It was concluded that the prevalence of infection found was probable due to intra-family transmission. Given the low probability of occurrence of a process of this type, the case grouping found is considered to be high.

Key words: Hepatitis B. Intrafamilial transmission. Asians immigrants. China. 


\section{INTRODUCCIÓN}

La hepatitis B es una enfermedad altamente endémica en regiones en desarrollo con densidades de población elevadas, como el sureste asiático, el África Subsahariana y la cuenca del Amazonas. En el año 2003, según datos de los Centros para Prevención y Control de Enfermedades (CDC) ${ }^{1}$, China se situó en la zona de alta prevalencia de hepatitis crónica B ( $\geq a 8)$, y España en la de prevalencia intermedia (2 a 7\%). En la actualidad se considera que más de 350 millones de personas en el mundo tienen hepatitis B crónica ${ }^{2,3}$. El virus es responsable de más de 300.000 casos de cáncer de hígado y de similar número de hemorragias gastrointestinales y ascitis ${ }^{3}$. La transmisión intrafamiliar está bien descrita y avalada con estudios del DNA del virus ${ }^{4-8}$. La vía perinatal es la principal en zonas de alta endemici$\mathrm{dad}^{2}$, sobre todo en hijos de madres con HBeAg positivo ${ }^{9}$, mientras que en zonas de baja endemicidad es la vía sexual ${ }^{2}$; aunque se han descrito otras vías de transmisión intrafamiliar, como la horizontal de padres a hijos o entre hermanos ${ }^{6,10}$, constituyendo la saliva una posible explicación de dicha transmisión ${ }^{11}$. Dado que en los recién nacidos un 95\% de la infección por el virus evoluciona hacia la cronicidad ${ }^{9}$, es necesario proteger a los hijos de madres portadoras con gammaglobulina y vacuna antihepatitis $\mathrm{B}^{12}$. En 1991 el Grupo Consultor Global del Programa de Expansión sobre Vacunación (EPI) de la Organización Mundial de la Salud (OMS) estableció metas para la introducción de la vacuna contra la hepatitis B en los programas nacionales de vacunación de todo el mundo. En mayo de 1992 la OMS avaló dicha recomendación ${ }^{13}$. España fue de los primeros países de Europa que implantó los programas de vacunación de hepatitis $\mathrm{B}^{14}$ pero en otros países, como China, las vacunas se generalizaron de forma más tardía.

En el año 2002 se detectaron dos personas con serologías positivas al virus de la hepati- tis B en miembros de las familias asiáticas de un municipio del Distrito Levante-Alto Almanzora, por lo que se inició un estudio de convivientes. El objetivo de la investigación fue conocer la amplitud de la infección en el entorno familiar de las personas con serologías positivas.

\section{SUJETOS Y MÉTODOS}

Se realizó un estudio observacional descriptivo en familias procedentes de China, de la provincia de Zhejiang, afincadas en España desde hacía años y residentes en un municipio del Distrito Levante-Alto Almanzora de Almería. Se analizaron las variables edad, sexo, diagnóstico serológico, factores de riesgo y actitud sanitaria. Se utilizó la información contenida en los siguientes sistemas de registro: historias clínicas de atención primaria y de hospital, datos serológicos de hepatitis B, encuesta epidemiológica y cartillas de vacunación.

Para la interpretación de los datos serológicos se utilizó la clasificación de los $\mathrm{CDC}^{1}$ (tabla 1) y se comprobó el estado vacunal de los niños mediante las cartillas de vacunación.

El estudio se inició en el año 2002 al detectarse una serología positiva para hepatitis B en una mujer embarazada en la consulta de Atención Primaria. La mujer era miembro de una familia de raza china que procedía de la zona de Zhejiang. Se realizó un estudio de contactos a todos los miembros de su familia, los cuales constituían una agrupación familiar extensa que a su vez estaba formada por tres familias que vivían en la misma casa. Posteriormente se detectó otra serología positiva en una mujer también embarazada que procedía de la misma zona, y que estaba relacionada con las familias anteriores, por lo que se amplió el estudio solicitándose serología para la hepatitis B a otras tres familias, consideradas otra agrupación familiar extensa ya que aunque vivían 
Tabla 1

Interpretación de serologías de hepatitis B. (fuente: CDC)

\begin{tabular}{|c|c|c|}
\hline Tests & Resultados & Interpretación \\
\hline $\begin{array}{c}\text { HBsAg } \\
\text { anti-HBc } \\
\text { anti-HBs }\end{array}$ & $\begin{array}{l}\text { negativo } \\
\text { negativo } \\
\text { negativo }\end{array}$ & Susceptible \\
\hline $\begin{array}{c}\text { HBsAg } \\
\text { anti-HBc } \\
\text { anti-HBs }\end{array}$ & $\begin{array}{l}\text { negativo } \\
\text { positivo } \\
\text { positivo }\end{array}$ & Inmune debido a infección natural \\
\hline $\begin{array}{c}\mathrm{HBsAg} \\
\text { anti-HBc } \\
\text { anti-HBs }\end{array}$ & $\begin{array}{c}\text { negativo } \\
\text { negativo } \\
\text { positivo }\end{array}$ & Inmune debido a vacuna \\
\hline $\begin{array}{c}\mathrm{HBsAg} \\
\text { anti-HBc } \\
\text { IgM anti-HBc } \\
\text { anti-HBs }\end{array}$ & $\begin{array}{l}\text { positivo } \\
\text { positivo } \\
\text { positivo } \\
\text { negativo }\end{array}$ & Infección aguda \\
\hline $\begin{array}{c}\mathrm{HBsAg} \\
\text { anti-HBc } \\
\text { IgM anti-HBc } \\
\text { anti-HBs }\end{array}$ & $\begin{array}{l}\text { positivo } \\
\text { positivo } \\
\text { negativo } \\
\text { negativo }\end{array}$ & Infección crónica \\
\hline $\begin{array}{c}\text { HBsAg } \\
\text { anti-HBc } \\
\text { anti-HBs }\end{array}$ & $\begin{array}{c}\text { negativo } \\
\text { positivo } \\
\text { negativo }\end{array}$ & Cuatro interpretaciones posibles* \\
\hline \multicolumn{3}{|c|}{$\begin{array}{l}\text { *1. Reconversión desde una forma aguda de infección HBV. } \\
\text { 2. Poco inmune y el test no es bastante sensible para detectar niveles muy bajos de anti- } \\
\text { HBs en suero. } \\
\text { 3. Falso positivo de anti-HBc. Susceptible. } \\
\text { 4. Niveles indetectables de HBsAg en suero. Portador. }\end{array}$} \\
\hline
\end{tabular}

en casas separadas residían en el mismo edificio y existían lazos de parentesco entre ellas. Todos los sujetos estudiados procedían de la misma zona de China y estaban afincados en España desde hacía varios años (entre cuatro y trece). Se incluyó a los 24 miembros de las familias en el estudio y se realizó un enfoque familiar para el abordaje. Se utilizó el genograma para representar a todos los sujetos, sus relaciones de parentesco, edades y resultados de las serologías solicitadas. Se realizó una captación activa de las personas estudiadas a través de tres miembros de las familias, los cuáles actuaron como intérpretes y enlaces en la transmisión de información. A todos ellos se les realizó una encuesta epidemiológica utilizando un cuestionario diseñado ad hoc, en el que se preguntó sobre síntomas relacionados con la enfermedad y factores de riesgo (transfusiones sanguíneas, uso de drogas vía parenteral, tatuajes, acupuntura, relaciones sexuales fuera de la pareja y uso compartido de algunos objetos en el domicilio, como cuchillas de afeitar, cepillos de dientes y agujas para perforar orejas).

Para el cálculo de probabilidad de ocurrencia del proceso a estudiar en las familias se utilizó la distribución binomial, considerando la prevalencia en el país de origen, China, aproximadamente en un $8 \%$.

\section{RESULTADOS}

La primera agrupación familiar estaba compuesta por 14 miembros y a su vez formada por las familias 1, 2 y 3 ; la segunda agrupación por 10 personas que a su vez constituían las familias 4, 5 y 6 . En las figuras 1 y 2 se 
Figura 1

Genograma de la primera agrupación familiar (grupo 1): familias 1, 2 y 3. Representación de lazos de parentesco, sexo, edad y serología de hepatitis B

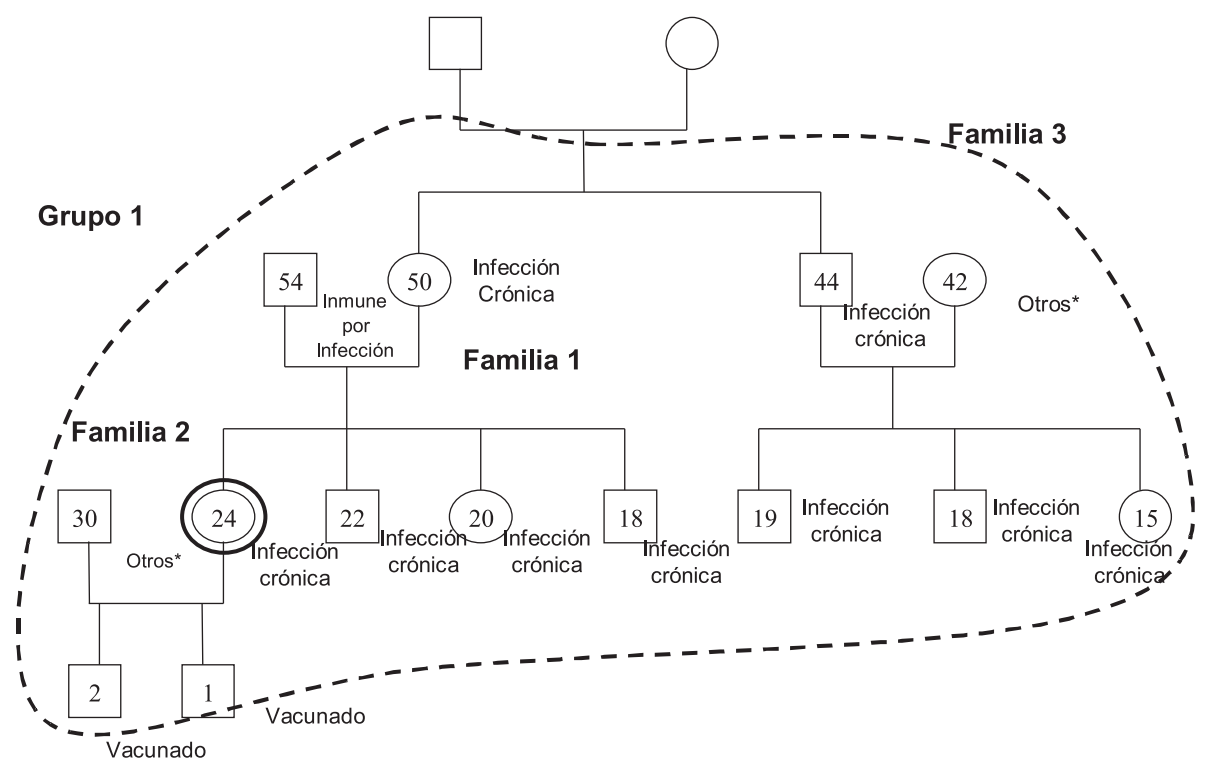

* Cuatro posibles interpretaciones según clasificación de los CDC. Ver Tabla 1.

Entre líneas discontinuas se representan los individuos que comparten domicilio.

\section{Figura 2}

Genograma de la segunda agrupación familiar (grupo 2): familias 4, 5 y 6. Representación de lazos de parentesco, sexo, edad y serología de hepatitis B

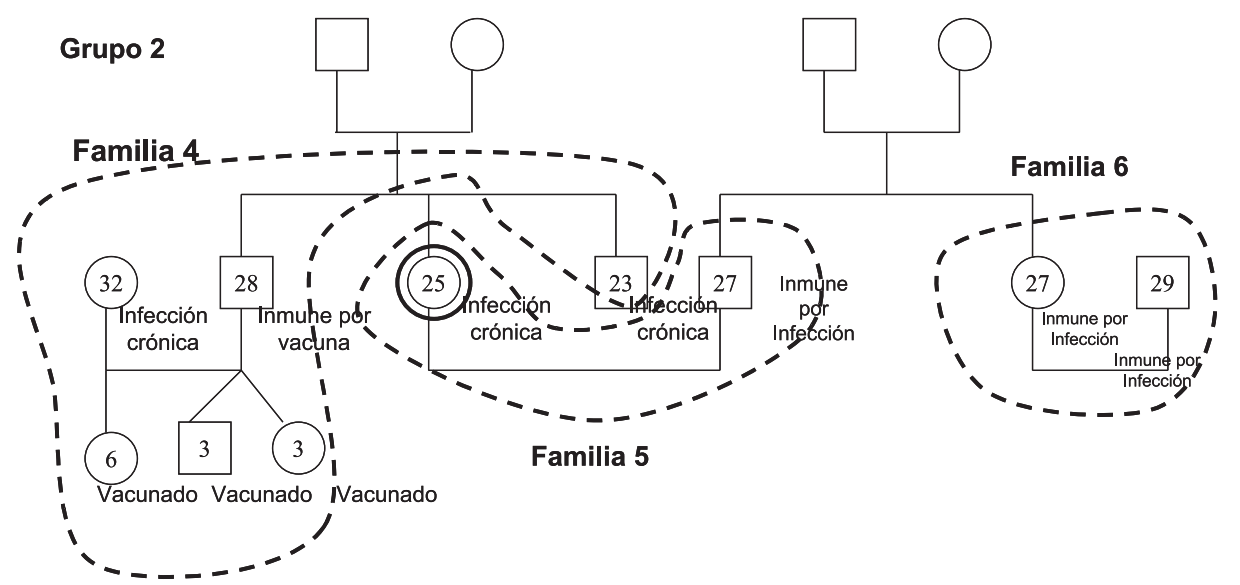

Entre líneas discontinuas se representan los individuos que comparten domicilio. 
representa mediante genogramas a todos los miembros de dichas agrupaciones, sus relaciones de parentesco, sexo, edad y resultados de las serologías realizadas.

De las 24 personas estudiadas el $58,3 \%$ eran varones y el $41,7 \%$ mujeres, con edades comprendidas entre 1 y 54 años (media de 23,4 y mediana de 23,5); el grupo de edad con más sujetos estudiados fue el de 21-30 años con el $37,5 \%$. Se detectaron 12 infecciones crónicas $(50 \%), 10$ de ellos además con $\mathrm{HBeAg}$ positivo (41,6\%), 5 niños nacidos en España estaban vacunados según el calendario vacunal; 2 adultos tenían anti$\mathrm{HBc}$ positivo y niveles bajos de anti-HBs (tabla 2). El 66,7\% de las personas con infecciones crónicas tenían entre 15 y 24 años.

$$
\left(\begin{array}{l}
24 \\
12
\end{array}\right) \times 0,08^{12} \times 0,92^{12}=0,000000066
$$

La probabilidad de ocurrencia de la agrupación de hepatitis crónicas encontrada fue de $0,066 \times 10^{-6}$.

En ningún caso se encontró relación con los factores de riesgo estudiados (transfusiones sanguíneas, uso de drogas vía parenteral, acupuntura, tatuajes, relaciones sexuales fuera de la pareja habitual, ni uso compartido de los objetos sobre los que se investigó). Todas las hepatitis crónicas se derivaron a la consulta de digestivo del hospital de referencia.

\section{DISCUSIÓN}

Se realizó un estudio familiar y se encontró un porcentaje elevado de hepatitis $\mathrm{B}$ en personas de raza china, con mayor número de casos en edades jóvenes de ambos sexos. En este sentido hay que tener en cuenta que China es considerada zona de alta prevalencia, ya que se han encontrado prevalencias elevadas en niños y adultos ${ }^{15}$. En otros países como el Reino Unido y Estados Unidos se ha encontrado mayor prevalencia de hepatitis crónica en población de raza china que en autóctona ${ }^{16,17}$. En Brasil se halló mayor prevalencia en personas de origen asiático ${ }^{18}$, y en Italia se encontró alta prevalencia en centros donde convivían niños de dicho país con otros procedentes de zonas con alta endemicidad $^{19}$. En España se han realizado estudios con inmigrantes de distintos paí$\operatorname{ses}^{20-24}$. Uno de ellos estimó prevalencia de HBsAg en mujeres asiáticas con edades entre 21 y 40 años en un $20 \%{ }^{20}$. En otros estudios se han encontrado prevalencias elevadas en inmigrantes procedentes de otros países como África Subsahariana, Latinoamérica y Europa del Este $20,22,24$.

Aunque se considera que la agrupación estudiada presentó un número elevado de

Tabla 2

Distribución de casos según sexo, edad y resultados serológicos

\begin{tabular}{|c|c|c|c|c|c|c|}
\hline Edad & \multicolumn{2}{|c|}{ Sexo } & \multicolumn{4}{c|}{ Serología } \\
\hline & $\mathrm{M}$ & $\mathrm{F}$ & $\begin{array}{c}\text { Inmune por } \\
\text { Infección natural }\end{array}$ & $\begin{array}{c}\text { Inmune } \\
\text { por vacuna }\end{array}$ & $\begin{array}{c}\text { Infección } \\
\text { crónica }\end{array}$ & $\begin{array}{c}\text { Otros } \\
\text { (Anti-HBc } \\
\text { positivo) }\end{array}$ \\
\hline $\mathbf{0 - 1 0}$ & 3 & 2 & & 5 & & \\
\hline $\mathbf{1 1 - 2 0}$ & 3 & 2 & & & 5 & 1 \\
\hline $\mathbf{2 1 - 3 0}$ & 6 & 3 & 3 & 1 & 4 & 1 \\
\hline $\mathbf{3 1 - 4 0}$ & & 1 & & & 2 & \\
\hline $\mathbf{4 1 - 5 0}$ & 1 & 2 & & & & $\mathbf{2}$ \\
\hline$>\mathbf{5 0}$ & 1 & & 1 & $\mathbf{6}$ & $\mathbf{1 2}$ & \\
\hline Total & $\mathbf{1 4}$ & $\mathbf{1 0}$ & $\mathbf{4}$ & & & \\
\hline
\end{tabular}


casos y que la probabilidad de que ocurra un proceso de estas características es muy baja, nuestros resultados están en consonancia con los descritos por otros autores que han encontrado porcentajes entre 66 y $81 \%$ de prevalencia de hepatitis crónica entre miembros de familias asiáticas ${ }^{18} \mathrm{y}$ otros estudios familiares que han documentado un porcentaje del $100 \%$ de infección entre los hijos ${ }^{10}$.

Dado el grado de parentesco entre los sujetos estudiados pensamos que se ha podido producir una transmisión intrafamiliar madre a hijo, o sexual entre algunos miembros, vías de transmisión también descritas por otros autores ${ }^{2,18,25}$ y que estaría corroborado por la bibliografía sobre estos tipos de transmisión de la enfermedad en China ${ }^{2,26}$. Pero con la encuesta epidemiológica realizada no se pueden descartar otras formas de transmisión intrafamiliar, como la horizontal padres a hijos o entre hermanos, descrita en distintos estudios ${ }^{4,10}$ en las que podría cobrar mucha importancia la saliva por la clara correlación encontrada entre el DNA del virus en suero y en la saliva ${ }^{11}$. Aunque este estudio se realizó en el año 2002 y los casos se detectaron en esa época, probablemente la infección fue adquirida en edades tempranas de la vida, como han indicado otros autores ${ }^{4}$, lo que podría explicar estos mecanismos de transmisión. Serían necesarios, sin embargo, otros estudios más específicos del DNA del virus en los distintos miembros de la familia para poder evidenciar todos estos mecanismos de transmisión, como se ha indicado en otros trabajos de la bibliografía consultada ${ }^{4-8}$.

Un aspecto a destacar es que la mayoría de los individuos estudiados, nacidos en China, con edades comprendidas entre 15 y 32 años, no se han beneficiado de programas de vacunación, ya que aunque en 1983 se iniciaron algunos programas en la zona de Qidong ${ }^{27}$, fue a partir de 1994 cuando el $97,5 \%$ de los puntos de vigilancia de enfermedad de las zonas urbanas y $73,9 \%$ de los rurales se incluyeron en el EPI, solicitándose serologías a las mujeres embarazadas y administrando a los recién nacidos dosis de vacunas y gammaglobulina en la mayoría de los puntos de vacunación ${ }^{28}$. Ha sido en los últimos años cuando se ha planificado integrar la vacuna en todo el territorio nacional de China $^{27}$ y se ha indicado la posibilidad de plantear programas de vacunación entre adolescentes con el fin de disminuir el riesgo $^{29}$. Sin embargo, sí se ha constatado que los sujetos nacidos en España estaban vacunados. En este sentido hay que tener en cuenta que España fue de los primeros países de Europa que junto con Albania, Bulgaria, Francia, Israel, Italia y Portugal habían implantado los programas de vacunación de hepatitis $\mathrm{B}^{14}$.

La elaboración del genograma facilitó la labor, ya que proporcionó una visión rápida e integrada de todos los miembros familiares estudiados (edad, relaciones de parentesco y problemas biomédicos), aspectos descritos por algunos autores ${ }^{30}$. También permitió orientar las hipótesis hacia la transmisión de la enfermedad dentro de las familias. Tanto para la búsqueda de casos como para la aplicación de las actuaciones terapéuticas y preventivas indicadas, ha sido muy útil el enfoque familiar. Aunque este abordaje ha sido más utilizado para pacientes crónicos, inmovilizados y terminales ${ }^{31}$, en nuestro caso ha permitido conocer la prevalencia de la enfermedad y aplicar las medidas de intervención adecuadas a la vista de los resultados, implicando en ellas a todos los miembros del grupo familiar. Realizar la captación a través de tres miembros de las diferentes familias ha permitido solventar las dificultades idiomáticas y las diferencias culturales de los sujetos afectados, y asegurar que todos los miembros familiares han sido valorados adecuadamente aplicando las medidas indicadas en cada caso. Se han podido realizar medidas de educación sanitaria, aportando documentos escrito a todos ellos. En los dos casos en los que no quedaba clara su situación por tener anti-HBc positivo y niveles muy bajos de anti-HBs se les administró la 
vacuna antihepatitis B; y los casos con infección crónica fueron derivados a la consulta de digestivo. Se les explicó la importancia de la enfermedad, ya que se comprobó que, como algunos autores han descrito ${ }^{32}$, el grado de conocimiento de la enfermedad en este colectivo es bajo.

Aunque el estudio se ha realizado en un ámbito familiar, con las limitaciones que ello supone, dados los resultados encontrados se considera necesario realizar estudios de hepatitis B y tener en cuenta esta enfermedad en población inmigrante procedente de zonas con alta endemicidad, sobre todo en los grupos de edad que no se hayan beneficiado de programas de vacunaciones, con especial énfasis en mujeres en edad fértil. Además se considera conveniente intensificar la vacunación en los grupos de riesgo, como ya han indicado algunos autores en España $^{20,23}$.

En resumen, se puede concluir que se ha detectado una agrupación de casos de hepatitis B en familias de raza china procedentes de una zona con alta prevalencia, probablemente debidos a transmisión intrafamiliar. Dada la baja probabilidad de ocurrencia de un proceso de estas características se considera que la agrupación de casos encontrada es elevada.

\section{BIBLIOGRAFÍA}

1. Viral Hepatitis B. Disponible en: http//www. cdc.gov/ncidod/diseases/hepatitis/b/index.htm. (citado el 4 de mayo de 2004).

2. Maddrey WC. Hepatitis B: An important public health issue. J Med Virol 2000; 61: 362-66.

3. Ching Lung Lai MD, Vlad Ratziu MD, Man-Fung Yuen MD, Thierry Poynard MD. Viral hepatitis B. The Lancet 2003; 362: 2089-94.

4. Zampino R, Lobello S, Chiaramonte M, VenturiPasini C, Dumpis U, Thursz M, et al. Intra-familial transmission of hepatitis B virus in Italy: phylogenetic sequence analysis and amino-acid variation of the core gene. J Hepatol 2002; 36: 248-53.
5. Dumpis U, Holmes EC, Mendy M, Hill A, Thursz M, Hall A, et al. Transmission of hepatitis B virus infection in Gambian families revealed by phylogenetic analysis. J Hepatol 2001; 35: 99-104.

6. Oon CJ, Chen WN, Goo KS, Goh KT. Intra-familial evidence of horizontal transmission of hepatitis $B$ virus surface antigen mutant G145R. J Infect 2000; 41: 260-4.

7. Thakur V, Kazim SN, Guptan RC, Malhotra V, Sarin SK. Molecular epidemiology and transmission of hepatitis B virus in close family contacts of HBV-related chronic liver disease patients. J Med Virol 2003; 70: 520-8.

8. Chakravarty R, Neogi M, Roychowdhury S, Panda CK. Presence of hepatitis B surface antigen mutant G145R DNA in the peripheral blood leukocytes of the family members of an asymptomatic carrier and evidence of its horizontal transmission. Virus Res 2002; 90: 133-41.

9. Codoñer Franch P. Hepatitis B. El virus, técnicas de diagnóstico, epidemiología, enfermedad y sus posibilidades evolutivas. An Pediatr 2003; 58: 478-81.

10. Erol S, Ozkurt Z, Ertek M, Tasyaran MA. Intrafamilial transmission of hepatitis B virus in the eastern Anatolian region of Turkey. Eur J Gastroenterol Hepatol 2003; 15 : 345-9.

11. Van der Eijk AA, Niesters HG, Gotz HM, Janssen HL, Schalm SW, Osterhaus AD, et al. Paired measurements of quantitative hepatitis B virus DNA in saliva and serum of chronic hepatitis B patients: implications for saliva as infectious agent. J Clin Virol 2004; 29: 92-4.

12. Shiraki K. Perinatal transmission of hepatitis B virus and its prevention. J Gastroenterol Hepatol 2000; 15 (Suppl): E11-15.

13. WHO Expanded Programme on Immunization. Hepatitis B control through immunization. Global programme for vaccines and immunization subcommittee meeting of the scientific advisory group of experts. Geneva, 12-16 June 1995.

14. Roure C. Overview of epidemiology and disease burden of hepatitis B in the European region. Vaccine 1995; 13 Suppl 1: S18-21.

15. Zhuo J, Tao G, Ebrahim SH, Wang S, Luo Z Wang $H$. The relationship of hepatitis $B$ virus infection between adults and their children in Guangxi Province, China. J Hepatol 2000; 33:628-31. 
16. Kawsar M, Goh BT. Hepatitis B virus infection among Chinese residents in the United Kingdom. Sex Transm Infect 2002; 78: 166-8.

17. Miller LC, Hendrie NW. Health of children adopted from China. Pediatrics 2000; 105:E76.

18. Ono-Nita SK, Carrilho FJ, Cardoso RA, Nita ME, da Silva LC. Searching for chronic hepatitics B patients in a low prevalence area-role of racial origin. BMC Fam Pract 2004; 5 : 7 .

19. Giacchino R, Zancan L, Vajro P, Verucchi G, Resti M, Barbera C, et al. Hepatitis B virus infection in native versus immigrant or adopted children in Italy following the compulsory vaccination. Infection 2001; 29: 188-91.

20. Romea Lecumberri S, Duran Pla E, Cabezos Otón J, Bada Aínsa JL. Situación inmunológica de la hepatitis B en inmigrantes. Estrategia de vacunación. Med Clin (Barc) 1997; 109: 656-60.

21. Lacalle Rodríguez-Labajo M, Gil Juberías G, Sagardui Villamor JK, González López E, Martínez Ruiz R, Orden Martínez B. Resultados de la aplicación de un examen de salud en población inmigrante. Aten Primaria 2000; 25: 634-8.

22. Vall Mayans M, Arellano E, Armengol P, Escribà JM, Loureiro E, Saladié P et al. Infección por el virus de la inmunodeficiencia humana y otras infecciones de transmisión sexual en inmigrantes de Barcelona. Enferm Infecc Microbiol Clin 2002; 20: 154-56.

23. Rodríguez C, Castilla J, del Romero J, Lillo A, Puig ME, García S. Prevalencia de infección por el virus de la hepatitis B y necesidades de vacunación en colectivos de alto riesgo. Med Clin (Barc) 2003; 121: 697-9.

24. Ramos JM, Pastor C, Masía MM, Cascales E, Royo G, Gutiérrez-Rodero F. Examen de salud en la población inmigrante: prevalencia de infección tuberculosa latente, hepatitis B, hepatitis C, infección por el VIH y sífilis. Enferm Infecc Microbiol Clin 2003; 21: 540-2.

25. Nguyen MH, Keeffe EB. Chronic hepatitis B and hepatitis C in Asian Americans. Rev Gastroenterol Disord 2003; 3: 125-34

26. Wang S, Peng G, Li M, Xiao H, Jiang P, Zeng N et al. Identification of hepatitis $B$ virus vertical transmission from fetus by direct sequencing. Southeast Asian J Trop Med Public Health 2003; 34: 106-113.

27. Sun Z, Ming L, Zhu X, Lu J. Prevention and control of hepatitis B in China. J Med Virol 2002; 67: 447 50 .

28. Zeng XJ, Yang GH, Liao SS, Chen AP, Tan J, Huang ZJ et al. Survey of coverage, strategy and cost of hepatitis B vaccination in rural and urban areas of China. World J Gastroenterol 1999; 5: 320-3.

29. Lee A, Cheng FF, Chan CS, Lau LC, Lo AS. Seroepidemiology and risk factors of positive hepatitis $\mathrm{B}$ surface antigen amongst Chinese adolescents. Asia Pac J Public Health 2001; 13: 30-5.

30. De la Revilla L, Fleitas L, Prados MA, De los Ríos A, Marcos B, Bailón E. El genograma en la evaluación del ciclo vital familiar natural y de sus dislocaciones. Aten Primaria 1998; 21: 219-24.

31. De la Revilla L, Espinosa Almendro JM. La atención domiciliaria y la atención familiar en el abordaje de las enfermedades crónicas de los mayores. Aten Primaria 2003; 31: 587-91.

32. Thompson MJ, Taylor VM, Jackson JC, Yasui Y, Kuniyuki A, Tu SP et al. Hepatitis B knowledge and practices among Chinese American women in Seattle, Washington. J Cancer Educ 2002; 17: 222-6. 